\title{
Towards quantitative analysis of STEM image contrast of interfaces and surfaces
}

\author{
S. Stemmer,* James M. LeBeau,* Scott D. Findlay,**, Adrian J. D’Alfonso***, and Leslie J. \\ Allen*** \\ * Material Department, University of California, Santa Barbara, CA 93106-5050, U.S.A. \\ ** Institute of Engineering Innovation, School of Engineering, The University of Tokyo, Tokyo, \\ 113-8656, Japan \\ *** School of Physics, University of Melbourne, Victoria 3010, Australia
}

High-resolution transmission electron microscopy (HRTEM) and scanning transmission electron microscopy (STEM) are widely applied in investigations of the atomic structure of interfaces. Interpretation of images in both techniques critically relies on comparisons with image simulations. In particular, the intensity of columns in high-angle annular dark-field (HAADF or Z-contrast) images in STEM is sensitive to thermal diffuse scattering (TDS) and Debye-Waller factors, which can be significantly modified at defects, interfaces or surfaces. Phase contrast and associated contrast reversals dominate HRTEM images. In the literature, quantitative comparisons in HRTEM have resulted in a large $(100-400 \%)$ discrepancy between theory and experiment, which has become known as the Stobbs factor [1]. In contrast, we have recently shown that in Z-contrast STEM, experiments and theory agree quantitatively, to within a few percent [2]. In this presentation, we demonstrate excellent agreement between simulated and experimentally observed image contrast in bright-field STEM (Fig. 1) [3]. The results show that STEM can provide quantitative imaging in both coherent and incoherent imaging modes. We will show specific examples of quantitative analysis of interfaces in this presentation.

Accurate and precise determination of the local sample thickness is critical for quantitative comparisons between image simulations and experiments of interfaces. Although several methods have been established, there is a clear need for a method to determine the local specimen thickness and tilt that is compatible with the thin specimens used in high-resolution STEM imaging and does not require changes in the optical conditions and sample orientation away from those used for imaging. In this presentation we show that by incoherently averaging convergent beam electron diffraction patterns that are produced by a finely focused, scanned STEM nanoprobe, a pattern develops that does not depend on lens aberrations and effective source size but remains highly sensitive to specimen thickness, tilt, and polarity. We show that these position averaged convergent beam electron diffraction (PACBED) patterns can be used to determine sample thicknesses to better than $10 \%$, for the same conditions and sample thicknesses as used in atomic resolution scanning transmission electron microscopy imaging (Fig. 2) [4]. In this presentation, we discuss the application of PACBED for the quantitative analysis of interfaces.

\section{References}

[1] M. J. Hytch, and W. M. Stobbs, Ultramicroscopy 53, 191 (1994); A. Howie, Ultramicroscopy 98, 73 (2004). 
[2] J. M. LeBeau, S. D. Findlay, L. J. Allen, and S. Stemmer, Phys. Rev. Lett. 100, 206101 (2008); J. M. LeBeau, S. D. Findlay, X. Wang, A. J. Jacobson, L. J. Allen, and S. Stemmer, Phys. Rev. $B$ 79, 214110 (2009).

[3] J. M. LeBeau, A. J. D’Alfonso, S. D. Findlay, S. Stemmer, L. J. Allen, Phys. Rev. B 80, 174106 (2009).

[4] J. M. LeBeau, S. D. Findlay, L. J. Allen, S. Stemmer, Ultramicroscopy 110, 118 (2010).

[5] The research at UCSB was supported by the U.S. National Science Foundation (grant number DMR-0804631) and the Department of Energy (DE-FG02-06ER45994).

Bright-field

\begin{tabular}{|c|}
\hline 0.00 \\
\\
$-5 \mathrm{~nm}$ \\
\\
0.06 \\
\hline
\end{tabular}

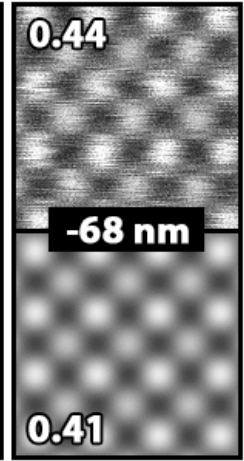

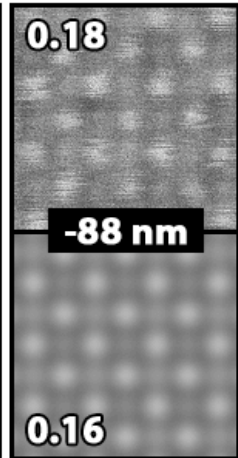

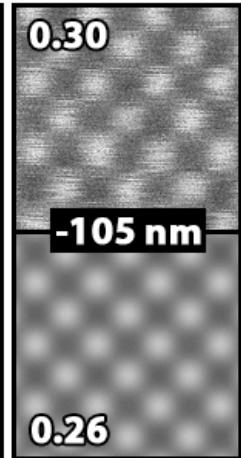

\section{(1)}

\section{Z-contrast}
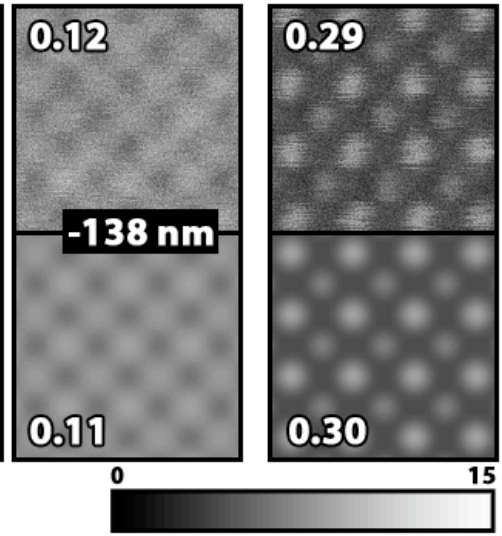

Percentage of incident probe (\%)

Figure 1: Left: experimental bright-field STEM images (top row in each column) compared to multislice absorptive model calculations (bottom row in each column). The upper labels in each image show their contrast values. The center (black background) labels state the defocus, with underfocus being negative. Right: experimental and simulated Z-contrast image. Note that all images are on an absolute intensity scale relative to the incident probe and reported as a fraction of the incident probe intensity (see intensity scale bar). The simulations have been convolved with a Gaussian of $0.11 \mathrm{~nm}$ FWHM to account for the effects of a finite source size.
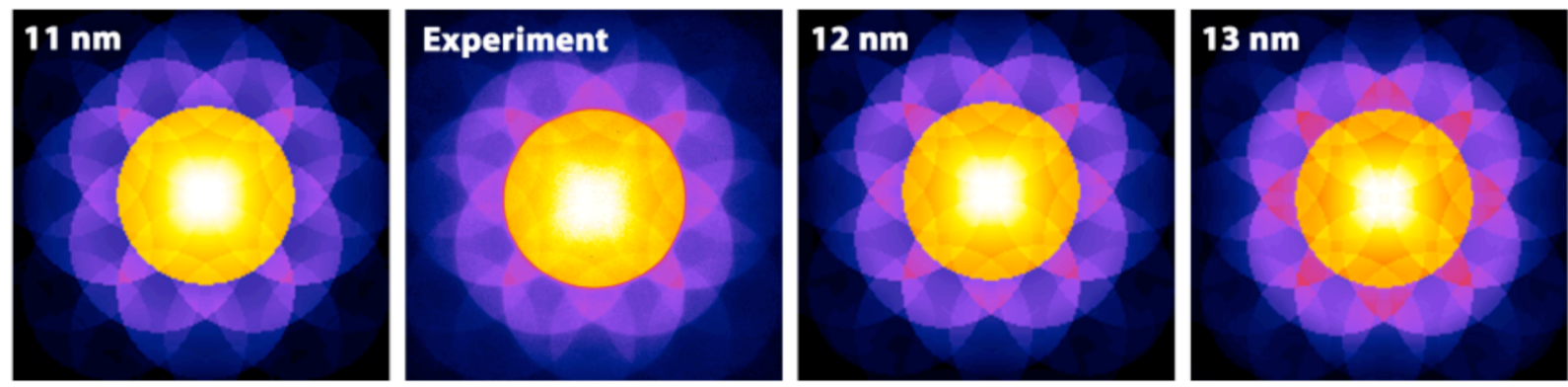

Figure 2: Comparison of an experimental PACBED pattern of $\mathrm{SrTiO}_{3}$ with simulated patterns for different thicknesses, simulated using the absorptive multislice and frozen phonon approaches. 